\title{
Simulation of HTTP-based Services Over LTE for QoE Estimation
}

\author{
Alessandro Vizzarri ${ }^{1} \quad$ Fabrizio Davide ${ }^{2}$ \\ ${ }^{1}$ Department of Enterprise Engineering, University of Rome Tor Vergata, Italy, \\ alessandro.vizzarri@uniroma2.it \\ ${ }^{2}$ Department of Innovation and Information Engineering, Guglielmo Marconi University, Italy, \\ f.davide@unimarconi.it
}

\begin{abstract}
Long Term Evolution (LTE) enables bandwidth consuming HTTP applications as video streaming. Mobile Network Operator (MNO) is committed to guarantee acceptable levels of Quality of Service (QoS) and Quality of Experience (QoE) perceived by the end user. A correlation between the transport informations with the application informations is an important approach to be adopted by the MNO. This correlation is more useful if a second entity, as the Over The Top (OTT), cooperates for the content delivery process. In the scientific literature different mathematical models are used in order to correlate QoE to the QoS. This paper aims at analyse them in case of of HTTP based Web services as HTTP web browsing and HTTP video streaming. Different scenarios are simulated using OPNET simulation software tool. They can differ if the service is fully managed by the MNO (MNO-managed class) or if OTT cooperates with own content (OTTmanaged). This is the case of YouTube. Results are analysed through regression k- means clustering techniques.
\end{abstract}

Keywords: LTE, QoS, QoE, over the top; YouTube; video streaming; key performance indicators

\section{Introduction}

In the last years, telecommunication technologies are enabling the delivery of bandwidth-consuming applications as web browsing or video streaming of several multimedia objects (Ericsson, 2008). The fourth generation of mobile networks, known as Long Term Evolution (LTE), is the first 3GPP cellular fully-IP standard. LTE is the most advanced technology to satisfy the increasing demand for mobile broadband services. It is able to offer to end users a download data rate up to $100 \mathrm{Mbps}$ and an upload data rate up to 50 Mbps (3GPP, 2007). LTE is also characterized by a flexible and interoperable fully-IP network architecture. We have also a direct management of Quality of Service (QoS) policies based on bearers and QoS Class Identifier (QCI). These informations are managed by Mobile Network Operators (MNOs) in order to efficiently deliver acceptable service levels to the endusers. QoS policies in LTE are mainly focused on measurable parameters called Key Performance Indicators (KPIs), namely bandwidth, delay, jitter, packet loss rate, data rate, priority. These QoS native features are crucial for an efficient network management of both data and voice services. Here we introduce an exercise of correlating the Quality of Experience (QoE) as perceived by the end user to the QoS as measured by the MNO at the network level for the HTTP web browsing and video streaming applications. We will introduce some relevant study cases, grouped in either MNO-managed class or OTT-managed class. We will review related works in Section II and we propose our approach in Section III. Section IV describes the study cases and present results from extensive simulations. Section V presents the mathematical models for the data which well describe in our cases the QoE vs QoS correlation. A final discussion states the application range of the proposed approach and its future improvements.

\section{Related Works}

Several mathematical models are proposed for QoE vs QoS correlation (M. Alreshoodi, et al, 2013). (H.G. Msakni et alii, 2013) presents the concept of Quality of Service (QoS) and Quality of Experience (QoE) applied to video quality assessment. (A. Vizzarri et al, 2013) present a review of studies on QoS in LTE networks. The Quality assessment methodology are essentially three: subjective, objective and network-based. In the first category, some authors introduce Mean Opinion Score (MOS) as a synthetic indicator of QoE, while network KPIs are assumed as indicators of QoS (ITU-T, 2003). (A. Vizzarri, 2014) analyzes the relationship between QoE in terms of MOS and QoS KPIs in case of Voice Over LTE (VoLTE) service. The objective assessment methodology focuses on the measurement of the signal as it would be perceived by an end user. Objective methods can be divided in three main groups: Full Reference (FR), Reduced Reference (RR) and No Reference (NR) (B. Wang et al, 2009). The FR method is based on the estimation of difference between the source video and the received video. The RR method analyzes a portion of informations extracted from the original video. The NR method predicts the video 
quality of the received video without accessing to the source video. The network assessment methodology gives an estimation of QoE on the basis of several QoS KPIs measured at network level. Network KPIs are essentially delay, jitter and packet loss rate (M. Siller, et al, 2003). In (M. Alreshoodi, 2013) a fuzzy-logic approach is proposed. (T.H Truong, 2012) analyzes the relationship between QoE and QoS in case of IPTV applications. Well known papers propose to express the QoE/QoS correlation through statistical analysis. The regression models more frequently used are: logarithmic (Weber-Fechner Law), exponential (IQX Hypothesis) and polynomial (S. Khorsandroo et al, 2012), M. Fiedler et al, 2010). In (J.Urrea , 2015) a multivariate statistical analysis has revealed the factors that influence the quality of a video streaming service on a Multi-hop Wireless Network. Relationships between the QoE and the QoS metrics have been identified via Multivariable Linear Regression (MLR) modeling. This methodology has supported the selection of the relevant factors and the reduction of the model dimensionality. A k-means clustering process allowed the authors to identify some operation ranges in terms of quality that relate the performances to KPIs.

\section{Proposed Approach}

\subsection{Methodology}

The methodology adopted for mapping QoE vs QoS in case of HTTP web browsing / video streaming is shown in Figure 1. It is to be remarked that we assume (coherently with some authors and differently from others):

- QoE metrics as Page Response Time (PRT), Video Response Time (VRT);

- QoS KPIs as end-to-end Delay (e2eDEL), Packet Loss Rate (PLR) and Throughput (THR).

\subsection{Network models}

The considered LTE network models are shown in Figure 2. The model is derived from LTE reference architecture and the related network nodes are the same as defined by 3GPP standard (3GPP, 2007), (3GPP, 2009). HTTP Web/Video Server is the content server containing both HMTL web pages and videos to deliver.

Depending on the owner of the contents to deliver (MNO or OTT), we distinguish for the sake of analysis two management cases for the HTTP web browsing and video streaming services: MNO-managed (class 1) and OTT-managed (class 2). In the first situation MNO is responsible for content ownership, service deliver and service transport. In the second situation MNO is only responsible for transport, while OTT is the content's owner and cooperates with MNO for service delivery. The two classes are shown in Figure 2.

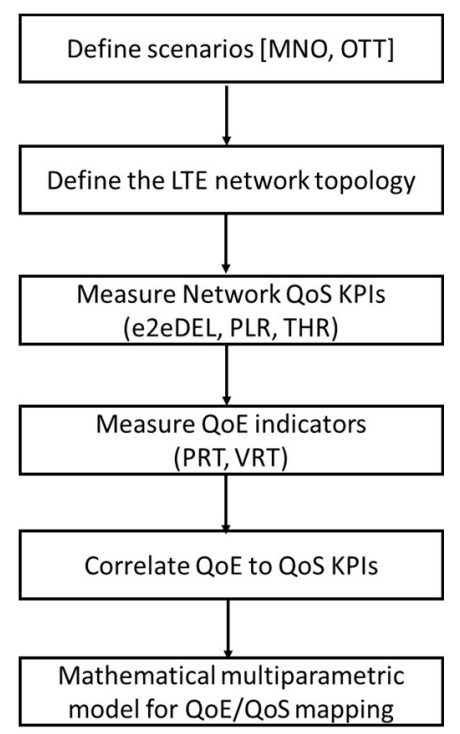

Figure 1. Workflow adopted for QoE/QoS correlation.

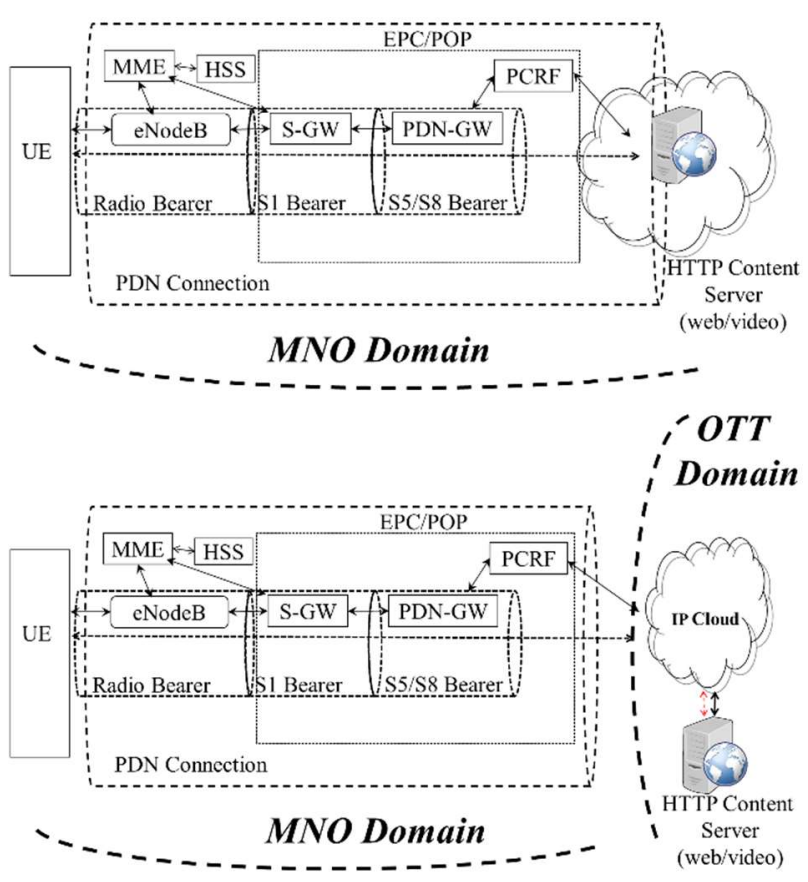

Figure 2. Workflow adopted for QoE/QoS correlation LTE service management classes: MNO-managed (uppermost), OTT-managed (lowermost).

\section{Simulation}

Network simulations have been performed using OPNET Modeler 17.5 PL6 software tool.

\subsection{OPNET Settings}

The User Equipment (UE) is modeled with $-1 \mathrm{dBi}$ Antenna Gain and $-200 \mathrm{dBm}$ receiver sensitivity. eNodeB bandwidth is $20 \mathrm{MHz}$ with 3 antenna sectors; Frequency Division Duplexing (FDD) is the Duplex mode, antenna gain of $15 \mathrm{dBi}$. As transmission model 
we chose a free space model. Evolved Packet System (EPS) bearer is characterized by a QCI equal to 6 (nonGuaranteed Bit Rate) with an Allocation Retention Priority (ARP) equal to 6. Simulation period is 3 minutes.

\subsection{Assumptions on users and networks}

On the end-user's side we have made a number of reasonable assumptions, as shown in Figure 3. The user sends a first request for a web page in HTML format. After 2 seconds starting from a download of complete web page, user sends a second request for a short video with duration of 20 seconds and a size around $1 \mathrm{MB}$. The HTML page is composed of: text $(180 \mathrm{~KB})$; a figure as top banner $(5 \mathrm{~KB})$; 30 small images $(7 \mathrm{~KB}$ each; 15 large images (20 KB each); a large image ( $35 \mathrm{~KB}$ ) as the initial video frame.

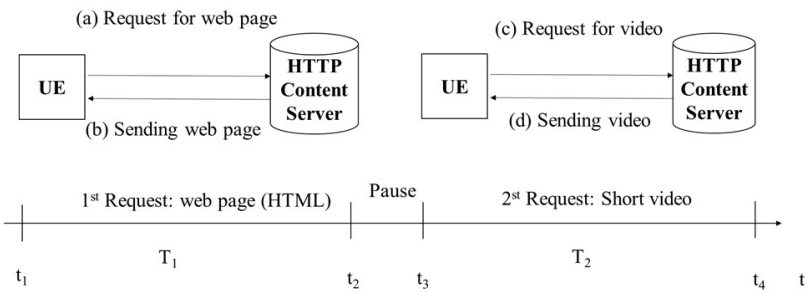

Figure 3. End-user's simulated activity.

On the network side we have modeled the two network models. In the network model of the first class the LTE network topology follows the following criteria:

- unique eNodeB and UE are located in the Rome area;

- EPC and HTTP server, managed by MNO, are located in the Milan area;

- Trunk link type is $1 \mathrm{Gbit} / \mathrm{s}$.

In the network model of the second class (OTTmanaged service), the LTE network topology is similar to the previous one but with some differences:

- HTTP server is a YouTube Primary Cache Server located in the Milan area. It is fully managed by You Tube, that plays the role of the OTT;

- The distance between the EPC and the HTTP web server is $100 \mathrm{~km}$;

- An IP cloud is considered, with additive $0.5 \%$ Packet Discard Ratio and 50ms Packet Latency. That to take into account impairments due to interconnection between the networks of MNO and OTT.

\subsection{Scenarios}

Fifteen study cases, called scenarios, are simulated for each class. Table 1 counts 30 scenarios, fifteen cases per class. Scenarios for class 2 are marked with $(*)$.
Table 1. Scenario Configurations - both MNO and OTT Management.

\begin{tabular}{|c|c|c|c|}
\hline \multirow[b]{2}{*}{ Scenario no. } & \multicolumn{3}{|c|}{ Features } \\
\hline & $\begin{array}{l}U E \\
n o .\end{array}$ & $\begin{array}{l}\text { S1 External } \\
\text { Traffic } \\
\text { Load }\end{array}$ & $\begin{array}{l}\text { Transmission } \\
\text { bitrate over } \\
\text { S1[bps] }\end{array}$ \\
\hline $\begin{array}{c}1,2,3 \\
16^{(*)}, 17^{(*)}, 18^{(*)}\end{array}$ & 1 & $0 \% ; 50 \% ; 75 \%$ & $\begin{array}{l}1,073,741,824 \\
536,870,912 ; \\
268,435,456\end{array}$ \\
\hline $\begin{array}{c}4,5,6, \\
19^{(*)}, 20^{(*)}, 21^{(*)}\end{array}$ & 5 & $0 \% ; 50 \% ; 75 \%$ & $\begin{array}{l}1,073,741,824 ; \\
536,870,912 ; \\
268,435,456\end{array}$ \\
\hline $\begin{array}{c}7,8,9, \\
22^{(*)}, 23^{(*)}, 24^{(*)}\end{array}$ & 10 & $0 \% ; 50 \% ; 75 \%$ & $\begin{array}{l}1,073,741,824 \\
536,870,912 \\
268,435,456\end{array}$ \\
\hline $\begin{array}{c}10,11,12, \\
25^{\left({ }^{*}\right)}, 26^{(*)}, 27^{(*)}\end{array}$ & 30 & $0 \% ; 50 \% ; 75 \%$ & $\begin{array}{l}1,073,741,824 \\
536,870,912 ; \\
268,435,456 \\
\end{array}$ \\
\hline $\begin{array}{c}13,14,15, \\
28^{(*)}, 29^{(*)}, 30^{(*)}\end{array}$ & 50 & $0 \% ; 50 \% ; 75 \%$ & $\begin{array}{l}1,073,741,824 \\
536,870,912 \\
268,435,456\end{array}$ \\
\hline $\begin{array}{c}1,2,3 \\
16^{(*)}, 17^{(*)}, 18^{(*)}\end{array}$ & 1 & $0 \% ; 50 \% ; 75 \%$ & $\begin{array}{l}1,073,741,824 \\
536,870,912 ; \\
268,435,456\end{array}$ \\
\hline $\begin{array}{c}4,5,6, \\
19^{(*)}, 20^{(*)}, 21^{(*)}\end{array}$ & 5 & $0 \% ; 50 \% ; 75 \%$ & $\begin{array}{l}1,073,741,824 \\
536,870,912 ; \\
268,435,456\end{array}$ \\
\hline $\begin{array}{c}7,8,9, \\
22^{(*)}, 23^{(*)}, 24^{(*)}\end{array}$ & 10 & $0 \% ; 50 \% ; 75 \%$ & $\begin{array}{l}\text { 1,073,741,824; } \\
536,870,912 ; \\
268,435,456 \\
\end{array}$ \\
\hline $\begin{array}{c}10,11,12, \\
25^{(*)}, 26^{(*)}, 27^{(*)}\end{array}$ & 30 & $0 \% ; 50 \% ; 75 \%$ & $\begin{array}{l}1,073,741,824 \\
536,870,912 \\
268,435,456\end{array}$ \\
\hline
\end{tabular}

\subsection{Results}

Table 2 shows the results of simulations for the study cases. Results for class 2 are marked with $(*)$.

\section{Linear Models Fit}

Multiple variable regression model is used for the identification of the relationship of QoE metrics Page Response Time (PRT), Video Response Time (VRT) with QoS KPIs, namely end-to-end Delay (DEL), Packet Loss Rate (PLR) and Throughput (THR). MNOmanaged and OTT-managed classes are separately analyzed. In order to assess the regression model, QoS KPIs are considered as variable predictors (independent variables), $\mathrm{QoE}$ is the corresponding response (dependent variable). Since the regression model with one variable predictor (one QoS KPI) and one response (QoE) gives unsatisfying scatter plot of residuals a Multivariate Linear Regression (MLR) model has been here applied. A generic MLR model is represented by

$$
Q o E=\beta_{0}+\beta_{1} * D E L+\beta_{2} * P L R+\beta_{3} * T H R+\varepsilon_{i}
$$

where $\beta_{0}, \beta_{1}, \beta_{2}, \ldots, \beta_{\mathrm{n}}$ are the unknown regression coefficients and $\mathscr{E} i \sim(\mathrm{N}, \sigma 2)$ is the error of each observed response. QoE as the response (dependent variable) may be one PRT or VRT, while DEL, PLR, THR are the independent variables. 
Table 2. Simulation Results.

\begin{tabular}{|c|c|c|c|c|c|}
\hline \multirow{2}{*}{$\begin{array}{l}\text { Scenar } \\
\text { io no. }\end{array}$} & \multicolumn{5}{|c|}{ Features Range [Min;Max] } \\
\hline & $\begin{array}{c}e 2 e \\
\text { Delay } \\
{[s]}\end{array}$ & $\begin{array}{c}P L R \\
{[\%]}\end{array}$ & $\begin{array}{c}L T E \\
T H R \\
{[M b p s]}\end{array}$ & $P R T$ & $V R T$ \\
\hline $\begin{array}{c}1,2,3 \\
16^{(*)}, 17^{(*)} \\
18^{(*)}\end{array}$ & $\begin{array}{c}{[0.08 ;} \\
0.09] ; \\
{[0.53 ;} \\
0.91] \\
\left.{ }^{*}\right)\end{array}$ & $\begin{array}{c}0.05 ; \\
0.91] \\
{[0.67 ;} \\
1.46] \\
\left.{ }^{*}\right)\end{array}$ & $\begin{array}{c}3.20 ; \\
3.30] ; \\
{[0.50 ;} \\
0.80] \\
\left.{ }^{*}\right)\end{array}$ & $\begin{array}{c}3.80 ; \\
4.18] ; \\
{[7.85 ;} \\
15.10] \\
\left({ }^{*}\right)\end{array}$ & $\begin{array}{c}{[5.28 ;} \\
5.90] ; \\
{[33.71 ;} \\
43.30] \\
\left({ }^{*}\right)\end{array}$ \\
\hline $\begin{array}{c}4,5,6, \\
19^{(*)}, 20^{(*)}, \\
21^{(*)}\end{array}$ & $\begin{array}{c}{[0.08 ;} \\
0.09] ; \\
{[0.32 ;} \\
0.58] \\
\left.{ }^{*}\right)\end{array}$ & $\begin{array}{c}0.25 ; \\
0.43] ; \\
{[6.14 ;} \\
13.08] \\
\left(^{*}\right)\end{array}$ & $\begin{array}{c}{[2.60 ;} \\
2.78] ; \\
{[0.48 ;} \\
0.63] \\
\left(^{*}\right)\end{array}$ & $\begin{array}{c}4.70 ; \\
4.73] ; \\
{[9.09 ;} \\
13.90] \\
\left({ }^{*}\right)\end{array}$ & $\begin{array}{c}{[8.73 ;} \\
9.83] ; \\
{[44.57 ;} \\
49.77] \\
\left(^{*}\right)\end{array}$ \\
\hline $\begin{array}{c}7,8,9, \\
22^{(*)}, 23^{(*)}, \\
24^{(*)}\end{array}$ & $\begin{array}{c}{[0.08 ;} \\
0.09] ; \\
{[0.31 ;} \\
0.61] \\
{ }_{(*)}^{*}\end{array}$ & $\begin{array}{c}{[0.18 ;} \\
0.57] ; \\
{[12.19 ;} \\
21,82] \\
{ }_{(*)}\end{array}$ & $\begin{array}{c}{[1.88 ;} \\
2.03] ; \\
{[0.65 ;} \\
0.73] \\
\left({ }^{*}\right)\end{array}$ & $\begin{array}{c}6.13 ; \\
6.25] ; \\
{[11,64 ;} \\
15,71] \\
\left.{ }^{*}\right)\end{array}$ & $\begin{array}{c}13.15 ; \\
13.25] ; \\
{[39.14 ;} \\
50.08] \\
{ }_{(*)}\end{array}$ \\
\hline $\begin{array}{c}10,11,12 \\
25^{(*)}, 26 \\
\left(^{(*)}\right. \\
27^{(*)}\end{array}$ & $\begin{array}{l}{[0.76 ;} \\
0.77] ; \\
{[2.52 ;} \\
3.34] \\
\left.{ }_{(*)}\right)\end{array}$ & $\begin{array}{c}0.27 ; \\
0.50] ; \\
{[16,88 ;} \\
22,99] \\
\left.{ }^{*}\right)\end{array}$ & $\begin{array}{c}1.00 ; \\
1.05] \\
{[0.43 ;} \\
0.60] \\
\left(^{*}\right)\end{array}$ & $\begin{array}{c}9.21 ; \\
9.63] \\
{[8.94 ;} \\
17.09] \\
\left(^{*}\right)\end{array}$ & $\begin{array}{c}30.80 ; \\
31.40] ; \\
{[40.20 ;} \\
53.55] \\
{ }_{\left({ }^{*}\right)}\end{array}$ \\
\hline $\begin{array}{c}13,14,15 \\
28^{(*)}, 29 \\
\left(^{(*)}\right. \\
30^{(*)}\end{array}$ & $\begin{array}{c}0.74 ; \\
0.82] ; \\
{[4.33 ;} \\
5.18] \\
\left(^{*}\right)\end{array}$ & $\begin{array}{c}{[11.34 ;} \\
13.35] ; \\
{[25,31 ;} \\
38,56] \\
\left(^{*}\right)\end{array}$ & $\begin{array}{c}0.57 ; \\
0.59] ; \\
{[0.53 ;} \\
0.70] \\
\left(^{*}\right)\end{array}$ & $\begin{array}{c}10.66 ; \\
12.41] ; \\
{[7.31 ;} \\
11.52] \\
\left.{ }^{*}\right)\end{array}$ & $\begin{array}{c}50.98 ; \\
52.95] ; \\
{[41.03 ;} \\
53.96] \\
\left({ }^{*}\right)\end{array}$ \\
\hline $\begin{array}{c}1,2,3 \\
16^{(*)}, 17^{(*)} \\
18^{(*)}\end{array}$ & $\begin{array}{c}0.08 ; \\
0.09] ; \\
{[0.53 ;} \\
0.91] \\
\left.{ }^{*}\right)\end{array}$ & $\begin{array}{c}0.05 ; \\
0.91] \\
{[0.67 ;} \\
1.46] \\
\left.{ }^{*}\right)\end{array}$ & $\begin{array}{c}3.20 ; \\
3.30] \\
{[0.50 ;} \\
0.80] \\
\left(^{*}\right)\end{array}$ & $\begin{array}{c}{[3.80 ;} \\
4.18] ; \\
{[7.85 ;} \\
15.10] \\
\left(^{*}\right)\end{array}$ & $\begin{array}{c}{[5.28 ;} \\
5.90] ; \\
{[33.71 ;} \\
43.30] \\
\left({ }^{*}\right)\end{array}$ \\
\hline $\begin{array}{c}4,5,6, \\
19^{(*)}, 20^{(*)}, \\
21^{(*)}\end{array}$ & $\begin{array}{l}{[0.08 ;} \\
0.09] ; \\
{[0.32 ;} \\
0.58] \\
\left.{ }_{(*)}^{*}\right]\end{array}$ & $\begin{array}{c}0.25 ; \\
0.43] ; \\
{[6.14 ;} \\
13.08] \\
\left(^{*}\right)\end{array}$ & $\begin{array}{c}2.60 ; \\
2.78] ; \\
{[0.48 ;} \\
0.63] \\
\left(^{*}\right)\end{array}$ & $\begin{array}{c}4.70 ; \\
4.73] ; \\
{[9.09 ;} \\
13.90] \\
\left.{ }^{*}\right)\end{array}$ & $\begin{array}{c}{[8.73 ;} \\
9.83] ; \\
{[44.57 ;} \\
49.77] \\
\left.{ }^{*}\right)\end{array}$ \\
\hline $\begin{array}{c}7,8,9 \\
22^{(*)}, 23^{(*)}, \\
24^{(*)}\end{array}$ & $\begin{array}{c}{[0.08 ;} \\
0.09] ; \\
{[0.31 ;} \\
0.61] \\
\left.{ }_{(*)}\right)\end{array}$ & $\begin{array}{c}{[0.18 ;} \\
0.57] ; \\
{[12.19 ;} \\
21,82] \\
\left(^{*}\right)\end{array}$ & $\begin{array}{c}{[1.88 ;} \\
2.03] ; \\
{[0.65 ;} \\
0.73] \\
\left({ }^{*}\right)\end{array}$ & $\begin{array}{c}6.13 ; \\
6.25] ; \\
{[11,64 ;} \\
15,71] \\
\left({ }^{*}\right)\end{array}$ & $\begin{array}{c}{[13.15 ;} \\
13.25] ; \\
{[39.14 ;} \\
50.08] \\
\left(^{*}\right)\end{array}$ \\
\hline $\begin{array}{c}10,11,12 \\
25^{(*)}, 26 \\
\left(^{*}\right) \\
27^{(*)}\end{array}$ & $\begin{array}{l}{[0.76 ;} \\
0.77] ; \\
{[2.52 ;} \\
3.34] \\
\left.{ }_{(*)}^{*}\right]\end{array}$ & $\begin{array}{c}{[0.27 ;} \\
0.50] ; \\
{[16,88 ;} \\
22,99] \\
\left.{ }^{*}\right)\end{array}$ & $\begin{array}{c}{[1.00 ;} \\
1.05] \\
{[0.43 ;} \\
0.60] \\
{ }_{(*)}^{*}\end{array}$ & $\begin{array}{l}{[9.21 ;} \\
9.63] ; \\
{[8.94 ;} \\
17.09] \\
{ }_{(*)}\end{array}$ & $\begin{array}{c}{[30.80 ;} \\
31.40] ; \\
{[40.20 ;} \\
53.55] \\
\left(^{*}\right)\end{array}$ \\
\hline $\begin{array}{c}13,14,15 \\
28^{(*)}, 29 \\
{ }^{(*)} \\
30^{(*)}\end{array}$ & $\begin{array}{c}{[0.74 ;} \\
0.82] ; \\
{[4.33 ;} \\
5.18] \\
\left(^{*}\right)\end{array}$ & $\begin{array}{c}11.34 ; \\
13.35] ; \\
{[25,31 ;} \\
38,56] \\
\left(^{*}\right)\end{array}$ & $\begin{array}{c}0.57 ; \\
0.59] \\
{[0.53 ;} \\
0.70] \\
\left(^{*}\right)\end{array}$ & $\begin{array}{c}10.66 ; \\
12.41] ; \\
{[7.31 ;} \\
11.52] \\
\left(^{*}\right)\end{array}$ & $\begin{array}{c}50.98 ; \\
52.95] ; \\
{[41.03 ;} \\
53.96] \\
{ }_{\left({ }^{*}\right)}\end{array}$ \\
\hline
\end{tabular}

$\left.{ }^{*}\right)$ Service managed by OTT

\subsection{MNO-managed class}

\subsubsection{PRT as the QoE Metric}

The estimated regression model is as in

$$
\begin{aligned}
P R T= & 9.357+2.368 * D E L+9.619 * P L R \\
& -1.749 * T_{H} R_{i}
\end{aligned}
$$

This MLR model provides a low Root Mean Squared Error (RMSE) value equal to 0.443 , an R-squared value equal to 0.982 and an Adjusted R-Squared value equal to 0.97 : i.e. quite a good fit. The overall $\mathrm{p}$-value is $6.15 \mathrm{e}$ 10. It allows us to reject the null hypothesis. Figure 4 shows relevant statistical indicators for this model: the fit looks actually good, while residuals are well distributed and adequately symmetric.
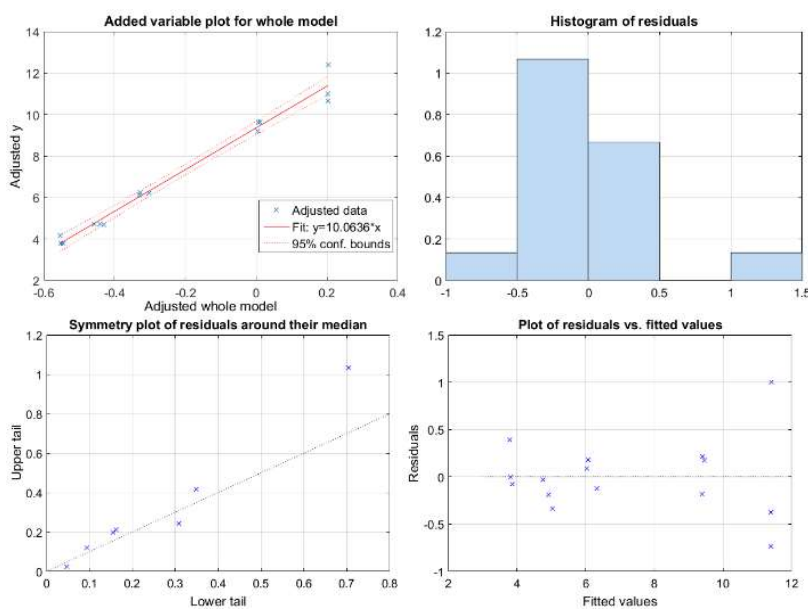

Figure 4. Statistical indicators for MLR of PRT in MNOmanaged class. From upper leftmost to right: plot for whole model vs samples; histograms of residuals; symmetry plot of residuals around their median; residuals vs fitted values.

\subsubsection{VRT as the QoE Metric}

The estimated regression model is as in

$$
\begin{aligned}
V R T= & 22.24+18.56 * D E L+148.7 * P L R \\
& -5.660 * T H R
\end{aligned}
$$

This MLR model provides a low Root Mean Squared Error (RMSE) value equal to 0.802 , an R-squared value equal to 0.998 and an Adjusted R-squared equal to 0.986 : i.e. quite a good fit. The overall p-value is $1.09 \mathrm{e}-$ 15. It allow us to reject the null hypothesis. Figure 5 shows relevant statistical indicators for this model: the fit looks actually good, while residuals are well distributed and adequately symmetric.
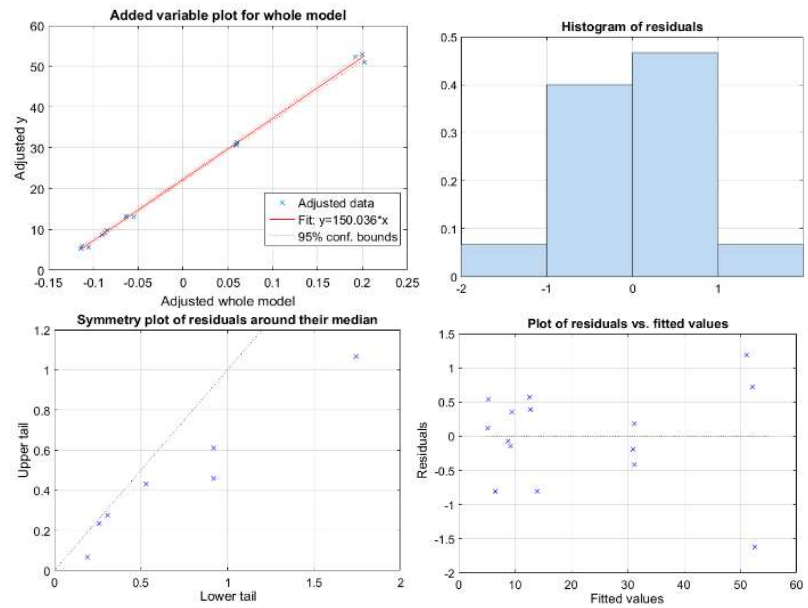

Figure 5. Statistical indicators for MLR of VRT in MNOmanaged class. From upper leftmost to right: plot for whole model vs samples; histograms of residuals; symmetry plot of residuals around their median; residuals vs fitted values. 


\subsubsection{Cluster Analysis for MNO-managed class}

Scenarios of the MNO-managed class, expressed in the 5 dimensional feature space, consisting of two QoE metrics and four QoS KPIs, have been clustered in three clusters, thanks to the well-known k-means technique [15]. Separation between clusters is fair as shown by the plot on the first two principal components (Figure 6).

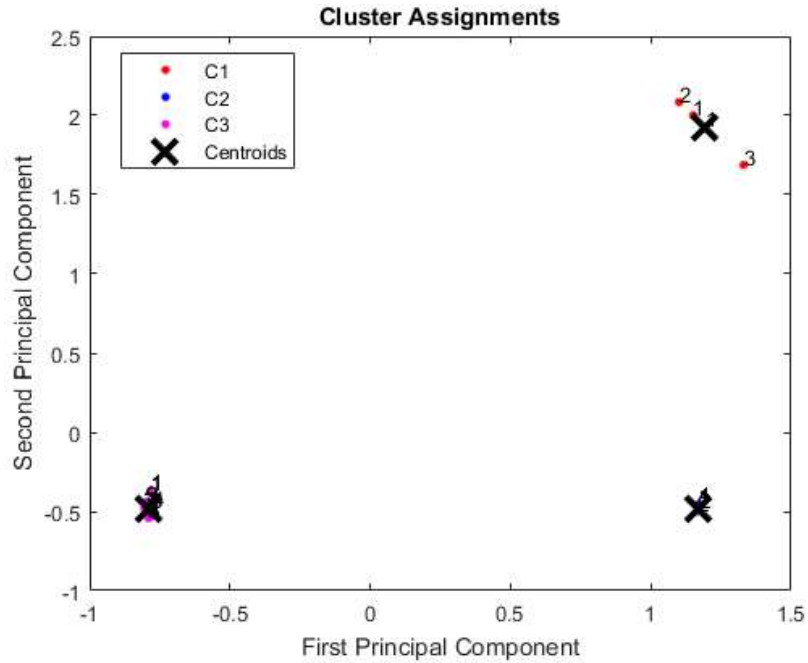

Figure 6. Clustering of scenarios for the MNO-managed class in the joint space of QoE metrics and QoS KPIs. Plot is on the plane of the first two principal components.

Meaning of the clusters is expressed in Table 3, which reports the position of the centroids of the three identified clusters in the 5-dim space. The quality of this clustering is evident from the changes of QoE between centroids: the VRT has the worst performance in $\mathrm{C} 1$, improves of $4.5 \mathrm{~dB}$ for $\mathrm{C} 2$, and $14.9 \mathrm{~dB}$ for $\mathrm{C} 3$. Same holds for PRT, though with lower gains (1.6 and 7.2 dB). As a conclusion, clustering well depicts differences between the scenarios in the MNO-managed class.

Table 3. Cluster Centroids generated by k-Means for MNO-managed Scenarios.

\begin{tabular}{|l|l|l|l|l|l|}
\hline $\begin{array}{l}\text { Cluster } \\
\text { Centroi } \\
\text { I Id. }\end{array}$ & $\begin{array}{l}\text { DEL } \\
{[s]}\end{array}$ & $\begin{array}{l}\text { PLR } \\
{[\%]}\end{array}$ & $\begin{array}{l}\text { THR } \\
{[M b p s]}\end{array}$ & $\begin{array}{l}\text { PRT } \\
{[s]}\end{array}$ & $\begin{array}{l}\text { VRT } \\
{[s]}\end{array}$ \\
\hline 1 & $\begin{array}{l}0.772 \\
4\end{array}$ & $\begin{array}{l}12.54 \\
\%\end{array}$ & 0.5783 & $\begin{array}{l}11.3 \\
6\end{array}$ & 52.05 \\
\hline 2 & $\begin{array}{l}0.764 \\
1\end{array}$ & $\begin{array}{l}0.360 \\
0 \%\end{array}$ & 1.025 & $\begin{array}{l}9.48 \\
9\end{array}$ & 31.01 \\
\hline 3 & $\begin{array}{l}0.083 \\
3\end{array}$ & $\begin{array}{l}0.370 \\
0 \%\end{array}$ & 2.650 & $\begin{array}{l}4.94 \\
4\end{array}$ & 9.339 \\
\hline
\end{tabular}

\subsection{OTT-managed class}

\subsubsection{PRT as the QoE Metric}

The estimated regression model is as in

$$
\begin{aligned}
P R T= & 7.347-0.813 * D E L+13.00 * P L R \\
+ & 6.796 * T H R
\end{aligned}
$$

This MLR model is affected by an excess Root RMSE at 3.11 and an insufficient R-squared value at 0.13 , while Adjusted R-Squared is meaningless (-0.08). The overall p-value is 0.591 . Thus we don't reject the null hypothesis. Let also remark that also the positive gain PRT/THR makes no sense. Figure 7 shows some evidences of the fact that the MLR model poorly describes the potential relationship QoE vs QoS in case of PRT.
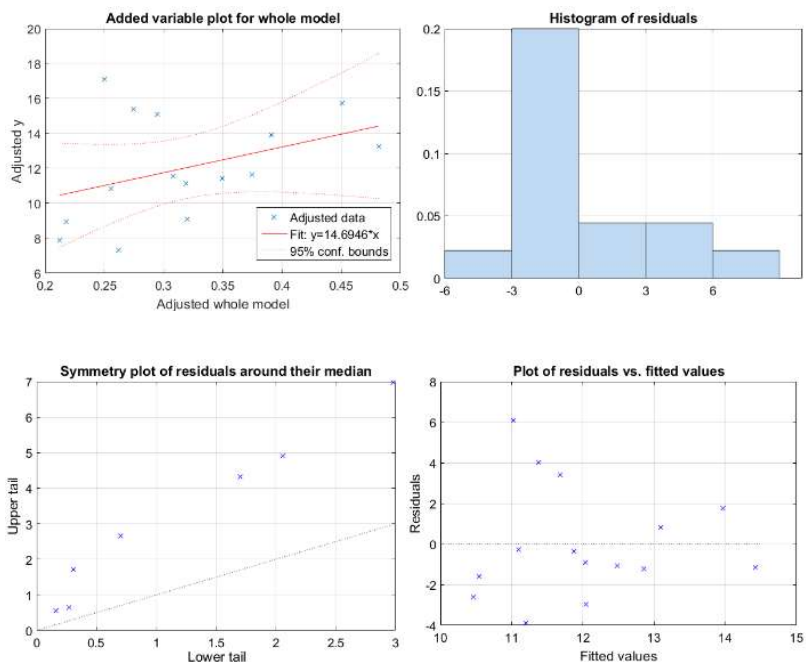

Figure 7. Statistical indicators for MLR of PRT in OTTmanaged class. From upper leftmost to right: plot for whole model vs samples; histograms of residuals; symmetry plot of residuals around their median; residuals vs fitted values.

\subsubsection{VRT as the QoE Metric}

The estimated regression model is as in

$$
\begin{aligned}
V R T= & 73.78-3.200 * D E L+32.46 * P L R \\
& -50.72 * T H R
\end{aligned}
$$

However, the MLR model is even worse than (4) ( $\mathrm{RMSE}=10.2$ and Adjusted R-Squared=0.14). The overall p-value of 0.211 makes the null hypothesis really likely. Figure 8 shows some evidences of the fact that the MLR model poorly describes the potential relationship QoE vs QoS in case of VRT.

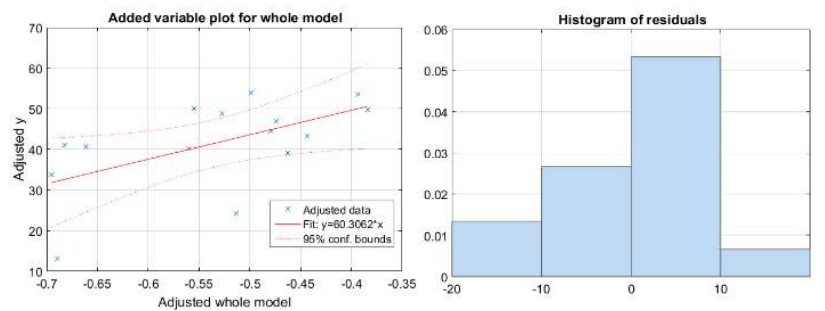



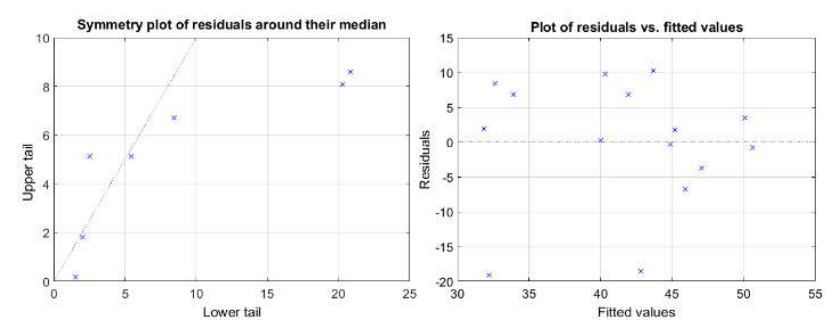

Figure 8. Statistical indicators for MLR of VRT in OTTmanaged class. From upperleftmost to right: plot for whole model vs samples; histograms of residuals; symmetry plot of residuals around their median; residuals vs fitted values.

\subsubsection{Cluster Analysis for OTT-managed class}

The statistical indicators in the OTT-managed class do not allow to take the fit as trusted. It means that a linear relationship between QoE KPIs and QoS metrics cannot really be ascertained. Cluster analysis can gives some more insight on relationships between scenarios of the OTT-Managed class, represented in the 5 dimensional feature space, consisting of two QoE metrics and three QoS KPIs. Figure 9 plots two clusters identified thanks to the k-means algorithm [15] on the plane identified by the first two principal components.

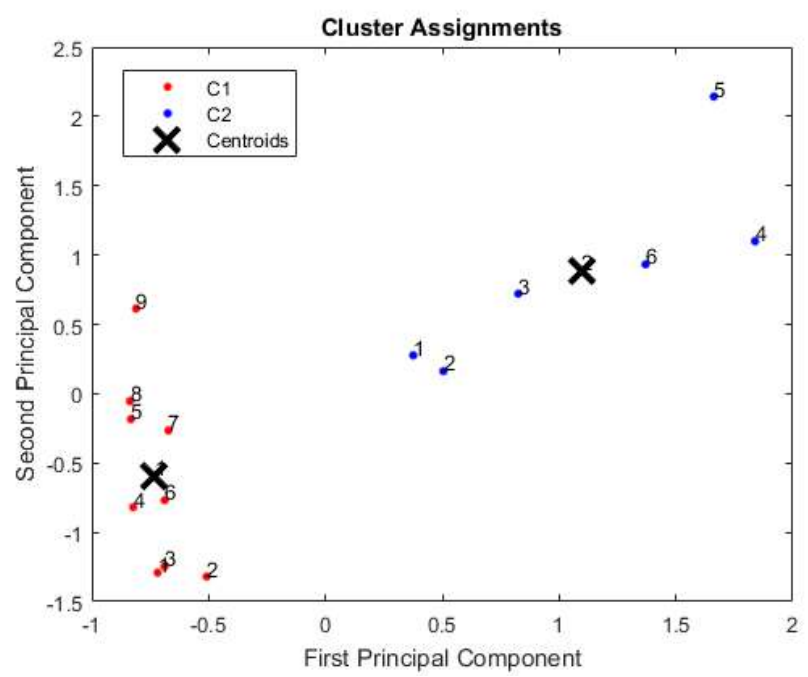

Figure 9. Identified clusters for the OTT-managed class including jointly both PRT and VRT as QoE metrics.

Separation between the two clusters is fair as per Figure 9. Though meaning of this separation is quite different as for the MNO-managed class. Table 4 reports the position of the centroids of the three identified clusters in the 5-dim space. It is straightforward noting that the two cluster centers are different as to the QoS KPIs, while they are nearly undistinguishable as for the QoE KPIs. Therefore, clustering reveals under a different perspective that QoE KPIs are not predictable starting from the QoS metrics.
Table 4. Cluster Centroids generated by k-Means for MNO-managed Scenarios.

\begin{tabular}{|l|l|l|l|l|l|}
\hline $\begin{array}{l}\text { Clu } \\
\text { ster } \\
\begin{array}{l}\text { Cen } \\
\text { troi } \\
\text { d Id. }\end{array}\end{array}$ & $\begin{array}{l}\text { DEL } \\
{[s]}\end{array}$ & $\begin{array}{l}\text { PLR } \\
{[\%]}\end{array}$ & $\begin{array}{l}\text { THR } \\
{[M b p s]}\end{array}$ & $\begin{array}{l}P R T \\
{[s]}\end{array}$ & $\begin{array}{l}\text { VRT } \\
{[s]}\end{array}$ \\
\hline 1 & 0.5026 & 8.610 & 0.6473 & 12.09 & 41.58 \\
\hline 2 & 3.829 & 24.84 & 0.5720 & 11.89 & 41.45 \\
\hline
\end{tabular}

\section{Discussion and Final Remarks}

The procedure of QoE metrics estimation out of QoS KPIs measured at network level is a consolidated process, and still a due attempt, regardless the complexity of networks and service architectures. Here we attempted for LTE networks the estimation of QOE vs QoS mapping thanks to MLR technique, with two distinct classes of scenarios. Our approach worked well as long as the MNO has been able to perform an end-toend management of the service delivered to the end user (MNO-managed class). It is possible to give a reliable estimation of QoE out of the QoS due to the MNO. This case may represent an incentive for OTT, who is supposed to have the ownership of contents, to cooperate with the MNO within the service delivery process. In the OTT-managed class the MNO loses the end-to-end control and the MLR technique turns out to give unsatisfactory results. In our understanding this is due to the fact that there is no-one with a full end-to-end management of the HTTP service.

Our results bring us to two key considerations. First, regression models are suitable for QoE vs QoS mapping if the QoS KPIs measured by MNO are the only factors that determine QoE. Second, if other factors out of the MNO's control affects the service delivery process (as the IP cloud considered in this work), the identification of the QoE vs QoS mapping comes to an unsatisfactory end.

A future step is to determine for the OTT-Managed class the minimal Service Level Agreement (SLA) of IP cloud that assures the existence of a QoE vs QoS (on the MNO side) mapping. This enables the OTT to negotiate with the MNO consistent QoS KPIs for the intended QoS metrics. Next steps regard how to assure that reliable mappings can be utilized on both sides, MNO and OTT, to deliver a quality-safe service to the enduser, within a sustainable business model. Impact of the QoE vs QoS mapping on costs and economic measures has to take part in the analysis

\section{References}

3GPP TS 23.401- 2007 GPRS Enhancements for E-UTRAN Access. Available via www.3gpp.org [accessed March 29, 2018]. 
3GPP TS 23.402-2009 Architecture enhancements for non$3 G P P$ Accesses. Available via www.3gpp.org [accessed March 29, 2018].

M. Alreshoodi and J. Woods. Survey on QoE/QoS correlation models for multimedia services. International Journal of Distributed and Parallel Systems, 3: 53-72, 2013.

M. Alreshoodi and J. Woods. An empirical study based on a Fuzzy Logic System to Assess the QoS/QoE Corelation for Layered Video Streaming. In Proceedings IEEE International Conference on Computational Intelligence and Virtual Environments for Measurement Systems and Applications, pages 180-184, 2013.

Ericsson Mobility Report. Ericsson Ltd, 2015.

ITU-T Recommendation, ITU-T Rec. P.800.1: Mean Opinion Score Terminology.

M. Fiedler and T. Hossfeld, and P. Tran-Gia. A Generic Quantitative Relationship between Quality of Experience and Quality of Service. IEEE Network, 2, pages 36-41, 2010 .

S. Khorsandroo and R. Md Noor, and S. Khorsandroo. Stimulus-Centric versus Perception-Centric Relations in Quality of Experience Assessment. In Proceedings IEEE Wireless Telecommunications Symposium, London, pages 1-6, 2017.

H. G. Msakni and H. Youssef. Is QoE estimation based on QoS parameters sufficient for video quality assessment?. In Proceedings 9th International Wireless Communications and Mobile Computing Conference, pages 538-544, 2013.

M. Siller and J. Woods. QoS arbitration for improving the QoE in multimedia transmission. In Proceedings IEEE International Conference on Visual Information Engineering, pages 238-241, 2003.

T.H Truong and Tai-Hung Nguyen, and Huu-Thanh Nguyen. On relationship between Quality of Experience and Quality of Service metrics for IMS-based IPTV networks. In Proceedings IEEE International Conference on Computing and Communication Technologies, Research, Innovation, and Vision for the Future, pages 1-6, 2012.

J.Urrea and N. Gaviria. Statistical performance evaluation of P2P video streaming on multi-hop wireless networks. In Proceedings 20th Symposium on Signal Processing, Images and Computer Vision, pages 1-6, 2015.

A. Vizzarri and S. Forconi. Review of Studies on End-to-End QoS in LTE Networks. In Proceedings AEIT Congress, pages 1-6, 2013.

A. Vizzarri. Analysis of VoLTE end-to-end quality of service using OPNET. In Proceedings European Symposium on Computer Modeling and Simulation, pages 452 - 457, 2014.

A. Vizzarri. Analysis of VoIP Over LTE End-To-End Performances in Congested Scenarios. In Proceedings Artificial, Intelligence, Modelling and Simulation, pages 393-343, 2014.

Wang, B. and Wen, X. and Yong, S., and Wei. A New Approach Measuring Users' QoE in the IPTV. In Proceedings IEEE Pacific-Asia Conference on Circuits, Communications and Systems, pages 453-456, 2009. 